\title{
Sostenibilidad económica del turismo en el Ecuador: Caso festividad de la "Mama Negra" Latacunga
}

\author{
Economic sustainability of tourism in Ecuador: the Latacunga "Mama \\ Negra" Festival case
}

Xavier Alejandro Silva Viteri. ${ }^{1}$, Luis Armijo Auquilla Belema. ${ }^{2}$, Lineth del Rocío Fernández Sánchez. ${ }^{3}$ \& Álvaro Andrés Auquilla Ordóñez. ${ }^{4}$

\begin{abstract}
In Ecuador, tourism development is presented as a sustainable alternative to replace state revenues that involve the exploitation of non-renewable resources; however, there is no strong evidence of economic sustainability. This research covers a series of ideas related to the economic sustainability of tourism, studying the case of the Black Mama Party in Latacunga. The objective of the work is to demonstrate if the studied tourist activity supports tourism in the territory. For this purpose, a bibliographic analysis was performed; and, a methodology was proposed that starts from the analysis of three years of festivities from 2017 to 2019, on the direct impact on the economy, analyzing the items and the investment spending made by the organizers. The indirect impact is measured with the data provided by the application of a survey to visitors, and the induced impact is established considering the two previous aspects, by means of the application of a multiplying factor on each indicator, to establish the amount of income approximate for the city of Latacunga in the established period. The results are satisfactory, since interesting income is observed for the city and its inhabitants; however, it is considered that these may be more significant if better planning is established, and the diversification

\footnotetext{
${ }^{1}$ Universidad Estatal Amazónica, Centro de Posgrado, Maestrante en Turismo, Mención Gestión del Turismo, Puyo-Ecuador, xas337@gmail.com

${ }^{2}$ Universidad Estatal Amazónica, Facultad Ciencias de la Vida, Carrera de Turismo, Puyo-Ecuador, luisauquilla10@gmail.com

${ }^{3}$ Universidad Estatal Amazónica, Facultad Ciencias de la Vida, Carrera de Turismo, Puyo-Ecuador, lineth1805@gmail.com

${ }^{4}$ Escuela Superior Politécnica de Chimborazo, Instituto de Posgrado y Educación Continua, RiobambaEcuador, andres.auquilla.ordonez@gmail.com
} 
of tourist activity is guaranteed, enhancing the infrastructure of the existing services. In addition, some indicators of the tourist profile are proposed that influence the economic sustainability of the tourist activity.

Key Words: Sustainability, Economy, Tourism, Festivity, Impacts

\section{Resumen}

En el Ecuador, el desarrollo turístico se presenta como una alternativa sostenible para sustituir ingresos estatales que implican la explotación de recursos no renovables; sin embargo, no existen evidencias contundentes de sostenibilidad económica. La presente investigación abarca una serie de ideas relacionadas con la sostenibilidad económica del turismo, estudiando el caso de la fiesta de la Mama Negra en Latacunga. El trabajo tiene por objetivo, demostrar si la actividad turística estudiada da soporte al turismo en el territorio. Para este fin, se realizó un análisis bibliográfico; y, se planteó una metodología que parte del análisis de tres años de festividades 2017 al 2019, sobre el impacto directo en la economía, analizando los rubros y el gasto en inversión que realizan los organizadores. Se mide el impacto indirecto con los datos proporcionados por la aplicación de una encuesta a los visitantes, y se establece el impacto inducido considerando los dos aspectos anteriores, para por medio de la aplicación de un factor multiplicador sobre cada indicador, establecer el monto de ingreso aproximado para la ciudad de Latacunga en el periodo establecido. Los resultados son satisfactorios, por cuanto, se observan ingresos interesantes para la ciudad y sus pobladores; sin embargo, se considera que, estos pueden ser de mayor cuantía si se establece una mejor planificación, y se garantiza la diversificación de la actividad turística, potenciando la infraestructura de los servicios existentes. Se propone, además, algunos indicadores del perfil del turista que influyen en la sostenibilidad económica de la actividad turística.

Palabras Clave: Sostenibilidad, Economía, Turismo, Festividad, Impactos

\section{Introducción}

Las ciudades históricas de interés patrimonial, sobre todo, aquellas que tienen la suerte de contar con festividades de relevancia socio cultural, en la actualidad son espacios de gran interés por parte de los agentes de desarrollo económico, considerando que ven en ellas un gran potencial para la generación de recursos alternativos en beneficio de las colectividades que las habitan. El turismo es un vehículo económico interesante para estos espacios territoriales, siempre que se realice con un enfoque en la sostenibilidad, basado en el respeto a la cultura indígena y mestiza, al medio ambiente y al patrimonio cultural tangible e intangible (Perea, Navarro, \& Luque, 2018). La sostenibilidad implica una relación racional entre el hombre y su entorno; los cambios que afectan el medio ambiente natural y la cultura imponen límites que deben analizarse antes de crear perturbaciones en el mismo (Ruíz \& García , 2014).

La aplicación de estrategias de análisis socio económico en el área del patrimonio cultural urbano para el turismo permite el estudio y la evaluación de las acciones creadas por la actividad de planificación, con miras a determinar, predecir, interpretar y comunicar los 
impactos que estas acciones causan en los entornos bajo las condiciones actuales, para alcanzar un modelo social con enfoques de sostenibilidad en el turismo (Blasco, Martínez, \& Alonso, 2017).

En este contexto, el impacto socio cultural en las ciudades involucradas en el turismo puede causar efectos singulares dentro de un territorio específico a lo que podría denominarse impactos potenciales que en última instancia son de importancia para el análisis del desarrollo turístico y su efecto en la economía local. Las características de los impactos mencionados aparecen expresadas en diferentes metodologías de evaluación, tales como: connotación, magnitud, importancia, sinergias, alcance y en casos excepcionales reversibilidad (Álvarez, 2015; Maldonado, Arúz, \& Pinos, 2017).

El termino impacto económico vinculado a la actividad turística aparece en la literatura especializada repetidamente e indica el cambio que la implementación de un proyecto inflige en un entorno sociocultural determinado, expresado en los resultados de su aplicabilidad (Castro, 2019; Eddy, 2016; Correra, 2015; Picornell, 2015). De acuerdo con otros autores, su importancia se interpreta en términos del bienestar, que, en sí, es lo que define al impacto en el desarrollo de los miembros de la comunidad.

En concordancia Güell (2019) y Castro (2018), argumentan que el impacto socioeconómico es la consecuencia directa e indirecta, beneficiosa (positiva) o adversa (negativa), que es producida por el hombre en los sistemas de convivencia social y de la que depende su desarrollo presente y futuro, como resultado de las transformaciones de la forma de vida y el intercambio comercial, causado por una acción o un conjunto de acciones de origen comunitario. El crecimiento urbano y el desarrollo poblacional en sus diferentes manifestaciones, así como las actividades recreativas o cualquier actividad de interrelación humana generan un impacto socioeconómico (Soto, 2015).

La evaluación del impacto socio económico al igual que la evaluación del impacto ambiental son herramientas que permiten la planificación de la inversión. Estas se perfeccionaron a través de los años de manera creativa facilitando la toma de decisiones, mejorando su implementación, determinando acertadamente sus alcances, todo ello, por intermedio de la gestión participativa, y valiéndose de planes, programas, políticas y estrategias para el desarrollo sostenible (Polanco, 2006).

Un proceso sistemático de la evaluación del impacto socioeconómico del turismo y la implementación de estrategias de gestión incorporan enfoques para el desarrollo sostenible en la concepción de los proyectos de inversión, teniendo en cuenta el futuro (Olivera, Cristobal, \& Saizar, 2016). Los propósitos de la evaluación de los impactos socio económicos como herramienta de gestión para el desarrollo sostenible consisten esencialmente en desarrollar información precisa y sistemática para diseñar estrategias en favor del desarrollo poblacional de un entorno determinado (Oyardi, Nazareno, Roldán, \& Ferrales, 2016).

La evaluación del impacto socio ambiental se inserta en este nuevo contexto, con un papel importante en su contribución como herramienta para el desarrollo sostenible, que incluye la predicción de los impactos en los entornos de forma integral y el análisis de la 
economía, la calidad de vida y la participación pública; además de los criterios tradicionales con respecto a la naturaleza exacta del proyecto y su ubicación (Güell, 2019).

\section{Medición económica de las fiestas populares}

La incidencia económica de los eventos y actividades culturales y sus repercusiones, relacionadas a determinadas manifestaciones festivas populares son susceptibles de ser medidas a través de estudios de impacto socio económico. Aunque su caracterización puede ser diversa, dependiendo que se trate de un caso u otro, el propósito fundamental de este tipo de trabajos es el de establecer cuantitativamente los efectos generados por las actividades culturales o festivas en un territorio determinado con duración temporal específica (Torres, Ullauri, \& Lalangui, 2018).

Estos trabajos de investigación, desde la perspectiva conceptual no son aparentemente complejos, sin embargo, en su realización, se encuentran dificultades de índole técnica que requieren de la consolidación de diversas fuentes de información, la aplicación de instrumentos para la adquisición de información de los diferentes actores y sobre todo de un análisis pormenorizado y cuidadoso que impida asumir sobre dimensionamiento de los efectos. En la actualidad la utilización de estas metodologías son tendencia en la investigación turística (Pearce, 2016).

Las investigaciones de impacto económico se caracterizan por asumir metodologías que, de manera general son comunes, sin embargo, se pueden encontrar diferencias en base a los flujos y factores en estudio. Considerando este argumento Herner (2016), establece que se encuentran comúnmente tres tipos de impacto que son susceptibles de ser medidos y estos son: los efectos directos que hacen referencia a los gastos ejecutados para el cumplimiento de actividades programadas por las instituciones o la institución organizadora en diversos rubros en el territorio y en un tiempo determinado.

Los efectos indirectos que son los diversos gastos que realizan los turistas que asisten al evento cultural o festivo, integrándose los servicios de hotelería, alimentación, transporte, compras diversas, localidades, entre otras; y, por último, los efectos inducidos que se refieren a todos aquellos aspectos que no se integran en las dos categorías anteriores, pero que se integran a la cadena de valor y fuera de los entornos en los que se realiza la festividad.

La caracterización de esta última categoría se presenta con características diferentes en función del tipo de estudio, en este sentido, mientras algunos investigadores se interesan en los efectos sobre las economías a nivel local, regional o nacional (Cuervo, 2017). Partiendo de un enfoque multiplicador y utilizando como referencia tablas de entradas y salidas, otros investigadores se interesan por aspectos más cualitativos (Picornell, 2015; Correra, 2015; Herrero, 2004). Como el desarrollo del capital humano del entorno social, el mejoramiento de la calidad de vida de los habitantes, el aparecimiento de actividades diferenciadas y la generación de puestos de trabajo, factores que por sus características son más difíciles de sistematizar y evaluar (Herner, 2016). 
El trabajo analizado sobre el cual se fundamente este artículo trata en lo posible de combinar los dos aspectos, pero limitando el análisis cualitativo debido a dos razones esenciales. En primer término, la utilización de una tabla de entrada y salida de información con características específicamente locales debido a la multiplicidad de factores externos que intervienen, esto obliga a establecer los impactos a través de fuentes diversas como la opinión de los administradores de los establecimientos de servicios sobre los efectos de la festividad, indicadores de ocupación, apreciaciones de gasto turístico, presupuesto del Gobierno Autónomo Descentralizado Municipal, entre otros. Por otro lado, se ha establecido la necesidad de emplear especificaciones de carácter operativo, para valorar las peculiaridades de un evento que se repite todos los años pero que cada vez presenta situaciones diferentes.

\section{Las fiestas tradicionales del Ecuador}

Siendo un fenómeno de carácter socio cultural las fiestas populares se manifiestan en una variedad de expresiones y por lo tanto dan lugar a diversas interpretaciones, dependiendo de la multiplicidad de manifestaciones sociales, étnicas y culturales de las parcialidades humanas que las celebran y de los participantes comunitarios que desempeñan diversos roles como también de las motivaciones emocionales, sociales y económicas que se generan en su entorno (Torres, Ullauri, \& Lalangui, 2018). Afirmando que, es real que las interpretaciones y significación sean susceptibles de ser interpretadas de diversas maneras (Proaño, 2019).

Las fiestas populares más importantes del Ecuador se incluyen en el calendario como un conjunto de celebraciones que se desarrollan durante todo el año y que se entretejen como una red de eventos que abarca a todo el territorio nacional. Las festividades populares pueden ser cívicas, religiosas, culturales, entre otras, se organizan cronológicamente por lugares, fechas o incluso periodos determinados. De tal manera que la calendarización de festividades responde al dónde, qué y cuándo, cuestionamientos que responden a los factores fundamentales para la definición e investigación de los eventos de celebración popular en los que se identifica de forma subjetiva o explicita la interacción entre los participantes en la celebración en un entorno específico y en un tiempo determinado (Escudero, 2017).

El calendario festivo revela no solo la diversidad de fiestas registradas, sino también la existencia de variaciones en la forma de celebrarlas, dependiendo de dónde se realicen y, por supuesto, de quiénes participen en ellas. Esto permite la visualización de las relaciones funcionales entre los actores que interactúan en las celebraciones y el roll que desempeñan en ellas (Herner, 2016).

La enumeración cronológica de las fiestas populares en el Ecuador se encuentra especificada de forma cronológica en el calendario festivo, el propósito fundamental en este tipo de trabajos es el de señalar los sitios y las fechas de las celebraciones, indicando la concentración de ellas en ciertos meses del año y haciendo notar la planificación y organización para la asignación de recursos y distribución del tiempo (Escudero, 2017). 


\section{La fiesta de la Mama Negra}

En la ciudad de Latacunga tradicionalmente entre el 23 y el 24 de septiembre de cada año se celebra de la fiesta de la Mama Negra conocida también como fiesta de la Capitanía, de la Virgen de las Mercedes o Santísima Tragedia. Aunque las autoridades de la sociedad civil oficializaron la celebración para el 11 de noviembre para festejar la independencia de la ciudad (Corrales, 2019).

Las dos fiestas comparten los personajes, diferenciándose en significación cultural porque la primera se fundamente en las raíces profundas de la tradición popular, mientras que la festividad de septiembre a pesar de ser más concurrida es una adaptación utilitaria de la tradición. La fiesta de la Mama Negra tiene sus raíces en la devoción de la Virgen de las Mercedes desde la época de la colonia y ha sido mantenida por la orden religiosa de los Mercedarios, doctrineros de gran influencia en la provincia de Cotopaxi. A lo largo del tiempo se ha convertido en una manifestación cultural que refleja la auténtica cultura popular y ha cobrado relevancia para el desarrollo turístico y económico de la localidad.

Los personajes de la Mama Negra tienen caracterización propia y son manifestaciones llenas de colorido y originalidad que, junto a las manifestaciones dancísticas, teatrales, poéticas, artesanales y gastronómicas generan una de las festividades más tradicionales de la cultura de la sierra ecuatoriana. Su valor y singularidad están expresados en el hecho de que siendo una fiesta que se desarrolla en el entorno urbano y que incorpora en sus visitantes la pluriculturalidad nacional, la Mama Negra, preserva muchos aspectos de los valores religiosos, mezclados con la cosmovisión indígena, que forjaron la ideología de los habitantes de Latacunga, en donde interactúan con un solo objetivo indígenas, mestizos, blancos, pobladores urbanos y suburbanos, hombres y mujeres de todas las edades y clases sociales (Chica, 2019).

En este contexto la festividad de la Mama Negra se proyecta como una manifestación de la sociedad y la cultura propia de la identidad de los latacungueños y los cotopaxenses. Se funde en una sola alegoría a las culturas afro, y blanco mestizo, otorgándole singularidad intercultural, que le ha permitido alcanzar la denominación de Patrimonio Cultural del Ecuador (Plasencio, 2015).

\section{Metodología}

El estudio de la sostenibilidad económica del turismo de la fiesta de la Mama Negra en la ciudad de Latacunga es el reflejo del impacto económico directo e indirecto que se realizó utilizando el método no experimental, observándose el fenómeno socio cultural relacionado a la generación de recursos económicos. Este estudio fue longitudinal, considerando que se obtuvieron los datos en tres periodos, en el presente caso se limitó al levantamiento de las variables de los periodos de septiembre y noviembre de los años 2017,2018 y 2019.

La fundamentación teórica de la investigación se extrae de la documentación existente en repositorios digitales y revistas científicas indexadas, en idioma español e inglés, proponiendo como elementos de búsqueda las variables en estudio: sostenibilidad 
económica del turismo y la festividad de la Mama Negra. Por otro lado, se obtuvo información de primera mano de los reportes de actividades e informes financieros de las organizaciones patrocinadoras del evento, sobre todo del GAD Municipal de la Latacunga, Ministerio de Turismo (MINTUR) y la empresa privada.

Para el análisis del impacto económico indirecto, los turistas fueron considerados en esta investigación como fuente de información primaria, pues, tienen patrones de consumo diferentes de los residentes y generan el ingreso de nuevo dinero circulante. Las encuestas se diseñaron específicamente para turistas que se hospedaron en los lugares de alojamiento, con un cuestionario enfocado a determinar un perfil turístico y sobre todo los indicadores de tiempo de estadía, gasto promedio de consumo diario, y destino de los rubros del gasto.

Para estudiar el impacto económico directo se consideraron como unidad de análisis (N1) los gastos registrados por el comité de fiestas del GAD Municipal de Latacunga, la Dirección Técnica del MINTUR, así como también, la información proporcionada por las empresas y auspiciantes que tramitaron el uso del espacio público en las instituciones pertinentes.

Como unidad poblacional de análisis para el estudio económico del impacto indirecto (N2), se consideraron 1.448 plazas de alojamiento de los prestadores de servicios de la ciudad de Latacunga, registrados en el catastro del Ministerio de Turismo (MINTUR, 2019).

La muestra para el análisis del impacto económico indirecto fue de 303 visitantes, en base a un criterio de muestreo aleatorio simple de un total de 1.448 plazas de alojamiento disponibles. Las entrevistas para lograr una medición completa se consideraron categoría de hospedaje y zonificación (Tabla 1), no se tomaron en cuenta en otros indicadores socioeconómicos o variables turísticas, la misma cantidad de plazas se mantienen para los tres años en estudio.

Tabla 1. Número de Encuestas por Establecimiento y Categoría

\begin{tabular}{lcccc}
\hline \multicolumn{1}{c}{ Tipo de } & Total & \multicolumn{3}{c}{ Categoría } \\
\cline { 3 - 5 } \multicolumn{1}{c}{ Establecimiento } & Encuestas & Primera & Segunda & Tercera \\
\hline Hostal & 77 & 25 & 27 & 25 \\
Hostal Residencial & 101 & 33 & 34 & 34 \\
Hostería & 7 & 7 & - & - \\
Hotel & 107 & 35 & 36 & 36 \\
Pensión & 11 & 3 & 4 & 4 \\
\hline Total, Encuestas & 303 & 103 & 101 & 99 \\
\hline
\end{tabular}

Fuente: Catastro MINTUR 2019

El factor de expansión para el impacto económico indirecto se calculó considerando el muestreo aleatorio simple que presenta iguales probabilidades de selección para cada 
individuo de la muestra, por tanto, el factor de expansión considera la cantidad de casos de la población equivalente a cada observación de la muestra (Cortés, 2008). Se utiliza entonces la siguiente fórmula.

$$
\text { fexp }=\frac{\begin{array}{c}
\text { Total de plazas ocupadas en los servicios } \\
\text { de alojamiento turístico }
\end{array}}{\text { Total de turistas que se alojaron por las }}
$$

\begin{tabular}{ccc}
\hline Año 2017 & Año 2018 & Año 2019 \\
f.exp $=\frac{3810}{241}$ & $f . \exp =\frac{3956}{245}$ & f.exp $=\frac{4203}{258}$ \\
$f . \exp =15,81$ & $f . \exp =16,15$ & f.exp $=16,29$ \\
\hline
\end{tabular}

De donde $f$ exp representa a un factor de expanción que se calcula tomando en cuenta la probabilidad de selección del turísta que llegó a la ciudad de Latacunga y pernoctó especificamente por motivo de la fiesta de la Mama Negra. Esta es una cifra que actua como un multiplicador llevando los datos muestrales a la población. Las pernoctaciones totales son el el número de turistas que ocuparón plazas en el servicio de alojamiento que, de acuerdo al MINTUR (2019) para el periodo 2017 fueron 3.810 con una muesta de 24, para el 2018 hubieron 3.956 con una muestra de 245, y para el 2019 alcanzaron 4.203 turistas con una tamaño de muestra de 258.

El analisis del impacto inducido se realizó utilizando la información obtenida del impacto económico directo, con base en la información conseguida de los informes de los gastos efectuados por los patrocinadores y organizadores oficiales de los eventos festivos; y, del impacto económico indirecto obtenido de la aplicación del muestreo a la que se le aplico un factor de expanción para inferir el total de la población en estudio.

El efecto inducido se aplicó en base al modelo Leontief (Montes \& Cantuche, 2005). El cual propone que, la proporción de factores utilizada por sector es constante, estableciendo de esta manera los coeficientes técnicos para los productos intermedios, utilizando los coeficioentes turisticos obtenidos de la tabla de entradas y salidas estimados para el trabajo de investigación.

Para la aplicación de las encuestas se realizó una zonificación, ubicando los servicios turisticos de hospedadje en los diferentes barrios de la ciudad de Latacunga, para el efecto se utilizó un mapa cartografico, en el cual se establecieron los sitios de acuerdo al catastro del MINTUR, se determinaron cuatro recorridos, y se conto con la participación de cuatro encuestadores en los dias 23 y 24 de septiembre; y, 10 y 11 de noviembre de 2019, fechas que corresponden a los principales eventos de la fiesta de la Mama Negra. 


\section{Resultados}

\section{Antecedentes de la sostenibilidad económica de la fiesta de la Mama Negra}

Las festividades de la Mama Negra en honor a la Virgen de la Merced representan para la ciudad de Latacunga un impulso comercial importante, las calles de la ciudad parecen transformarse por la presencia de visitantes durante los meses de septiembre y noviembre. En este espacio de interacción cultural festiva se forman grupos de amigos, vecinos, familias y priostes para celebrar. Destinándose para el efecto entre 3.000 a 10.000 dólares por grupo para financiar las actividades programadas y, conmemorar la representación religiosa y la fiesta de la independencia de Latacunga. El dinero aportado se invierte en la contratación de bailarines, bandas de música, transporte, alimentación, vestimentas, obsequios, entre muchos otros rubros que permiten armar el complejo entramado de la celebración.

El acto principal de la festividad es la cabalgata de la Mama Negra, realizada por los devotos y comerciantes de los mercados de El Salto Cerrado y La Merced. Estas actividades se realizan el 23 y 24 de septiembre la celebración religiosa, y el 9 de noviembre el desfile organizado por la municipalidad que es más de orden cívico (Vaca, 2016). De acuerdo con la información proporcionada por el GAD Municipal de Latacunga, en estos tres días participan en los festejos más de 500 personas como participantes directos, y se tiene la presencia de un promedio de entre 50.000 a 300.000 visitantes dependiendo de la fecha.

En la ciudad de Latacunga existen unos 40 sastres y costureras que se dedican a la confección de los atuendos característicos de los personajes que conforman el cortejo de la Mama Negra, otros locales alquilan la vestimenta y accesorios necesarios. Se estableció a través de encuesta que el alquiler de un traje puede costar desde 15 hasta 40 dólares diarios y, sin embargo, los locales ubicados en las calles céntricas de la ciudad de Latacunga no logran satisfacer la enorme demanda, al punto que, para alquilar una prenda, confeccionarla o bordarla con los nombres de los priostes o con alegorías de los personajes, los clientes suelen acudir con por lo menos tres meses de anticipación.

Es interesante destacar que la festividad asegura la participación en cada pregón de por lo menos 50 bandas de pueblo, danzas y ashangueros de la localidad o, traídos de otros sitios dentro de la provincia e incluso fuera de ella. En este sentido es común observar danzas y bandas de otras provincias de las tres regiones del Ecuador continental.

La Ashanga o canasta, característica gastronómica de la festividad, que contiene un cerdo adornado con cuyes, gallinas, botellas de licor, dulces, frutas y otros menjurjes, más tres cargadores y cinco negros loeros cuesta 900 dólares. La banda de músicos conformada por 18 integrantes tiene un valor de 800 dólares, y el grupo de danza de 16 bailarines con la vestimenta completa, dos camisones, tres huacos y un soplador cobra 500 dólares (Zambrano, 2015). 
Entre las actividades que se relacionan al festejo están la serenata a la imagen de la Virgen de las Mercedes que se efectúa afuera de la Iglesia las vísperas a la festividad, acto en el que los priostes realizan una inversión de aproximadamente 7.000 dólares. Los confites denominados "rompe muelas" o dulces de San Sebastián son tradicionalmente ofrecidos por los priostes, estos dulces son elaborados artesanalmente y se comercializan en el mercado Cerrado a 1,40 dólares la libra, generalmente una familia latacungueña adquiere unas cinco libras a un costo de 1,40 dólares para repartir en el desfile, estos dulces forman parte de los agrados que la municipalidad entrega en las jochas a los personajes que desfilan y a los visitantes (GAD Municipal de Latacunca , 2016).

La inversión del Cabildo y las instituciones patrocinadoras se aproxima a los 200.000 dólares, reportándose una ganancia de aproximadamente 2 millones de dólares en los tres días de las dos festividades. La representación de los personajes principales de la Cabalgata de la Mama Negra son personas representativas de la ciudad de Latacunga y que gozan de alta estima y representatividad de la comunidad, estos piden ayuda a familiares y amigos para costear la parte de la festividad que les corresponde asumir y, además gestión la consecución de recursos buscando patrocinadores que estén dispuestos a invertir en la festividad (Giacomotti, 2019).

Los datos anteriormente expuestos son algunos de los elementos y factores que intervienen en la realización de las festividades de la Mama Negra en la ciudad de Latacunga $y$, que de una u otra manera contribuyen para dar sostenibilidad a la celebración a través de la generación de actividades económicas, haciendo posible que muchas personas encuentren en este acto socio cultural la oportunidad para mejorar sus ingresos económicos, al menos durante este periodo de tiempo.

Los rubros mencionados en estos antecedentes son complejos de cuantificar, por efecto de la informalidad comercial con la que se realizan, pero lo rescatable de ellos es que forman parte de la tradición y patrimonio de la festividad.

\section{Impactos económicos directos}

Con el propósito de obtener la información de los gastos realizados por los financistas de la fiesta de la Mama Negra, los datos fueron homologados considerando la Clasificación Internacional Industrial Uniforme (CIIU) (Herrero, 2004). En donde se contemplan las siguientes funciones:

$$
\mathrm{IED}=\mathrm{GCF}+\mathrm{RDI}+\mathrm{GDT}
$$

Donde:

IED = Impacto Económico Directo; GCF = Gastos devengados por el comité de fiestas de la Mama Negra; RDI = Registro de Desembolsos de Inversores; GDM = Gastos devengados por el MINTUR.

En la tabla 2, se propone el consolidado de los años 2017, 2018 y 2019, en los que se analizan para cada periodo las funciones antes mencionadas. 
Tabla 2. Consolidación del gasto directo de la fiesta de la Mama Negra

\begin{tabular}{|c|c|c|c|c|c|}
\hline \multirow{2}{*}{$\begin{array}{l}\text { Descripción de la sección de } \\
\text { Casto }\end{array}$} & \multirow[t]{2}{*}{ Año 2017} & \multirow[t]{2}{*}{ Año 2018} & \multirow[t]{2}{*}{ Año 2019} & \multicolumn{2}{|c|}{ Total } \\
\hline & & & & Monto & $\%$ \\
\hline $\begin{array}{l}\text { Actividades artísticas, de } \\
\text { entretenimiento y recreativas }\end{array}$ & $64.599,08$ & 70639,68 & 89451,72 & $224.690,48$ & 65,54 \\
\hline $\begin{array}{l}\text { Actividades de alojamiento y } \\
\text { de servicio de comidas }\end{array}$ & $1.067,9$ & 1135,3 & 1431 & $3.634,2$ & 1,06 \\
\hline $\begin{array}{l}\text { Actividades de servicios } \\
\text { administrativos y de apoyo }\end{array}$ & $1.794,71$ & 1910,83 & 2477,58 & $6.183,12$ & 1,80 \\
\hline $\begin{array}{l}\text { Actividades financieras y de } \\
\text { seguros }\end{array}$ & 134,83 & 143,6 & 180 & 458,43 & 0,13 \\
\hline Actividades inmobiliarias & $5.058,65$ & 5347,05 & 6770,35 & $17.176,05$ & 5,01 \\
\hline $\begin{array}{l}\text { Administración pública y } \\
\text { defensa; planes de seguridad } \\
\text { social de afiliación obligatoria }\end{array}$ & $1.819,58$ & 1937 & 2446,37 & $6.202,95$ & 1,81 \\
\hline $\begin{array}{l}\text { Comercio al por mayor y al } \\
\text { por menor; reparación de } \\
\text { vehículos automotores y } \\
\text { motocicletas }\end{array}$ & 352,87 & 375,47 & 475,48 & $1.203,82$ & 0,35 \\
\hline Industrias manufacturas & $20.959,36$ & 20503,86 & 25953,2 & $67.416,42$ & 19,67 \\
\hline $\begin{array}{l}\text { Información y } \\
\text { comunicaciones }\end{array}$ & $3.754,34$ & 3996,46 & 5017,77 & $12.768,57$ & 3,72 \\
\hline Transporte y almacenamiento & 888,05 & 967,08 & 1216,33 & $3.071,46$ & 0,90 \\
\hline Total & $100.429,37$ & $106.956,33$ & $135.419,8$ & $342.805,5$ & 100,00 \\
\hline
\end{tabular}

Fuente: Comité Permanente, Empresas Privadas, MINTUR

Los gastos realizados para la fiesta de la Mama Negra obtenidos del comité de fiestas, desembolsos de inversores y gastos devengados por el MINTUR para la festividad, reflejan un aumento progresivo desde el año 2017 al año 2019, pero no es significativo, el monto total de gastos directos alcanzados fue de 342.805,50 dólares. Las áreas de gasto con mayor inversión de recursos fueron las relacionadas con las actividades artísticas de entretenimiento y recreativas, que para los tres años alcanzo un monto de 224.690,40 dólares; es decir, un $65,54 \%$, seguido por la inversión de la industria manufacturera con 67.416,42 dólares, que corresponde al 19,67\%; en tercer lugar, se encuentran las actividades inmobiliarias con 17.176,05 dólares, que representa el 5,01\%; las otras actividades se prorratean proporcionalmente, ver tabla 2.

Tabla 3. Gasto directo por el lugar de destino de inversión en la fiesta de la Mama Negra

\begin{tabular}{ccccccccc}
\hline \multirow{2}{*}{ Ciudad } & \multicolumn{2}{c}{ Periodo 2017 } & \multicolumn{2}{c}{ Periodo 2018 } & \multicolumn{2}{c}{ Periodo 2019 } & \multicolumn{2}{c}{$\begin{array}{c}\text { Total, tres } \\
\text { periodos }\end{array}$} \\
\cline { 2 - 9 } & Monto & \% & Monto & \% & Monto & \% & Monto & \% \\
\hline Latacunga & $58.249,03$ & 58 & $54.547,72$ & 51 & $77.189,28$ & 57 & $189.986,03$ & 59,30 \\
\hline
\end{tabular}




\begin{tabular}{lrcrcrccc}
\hline Otros Países & $11.047,23$ & 11 & 14.973 .85 & 14 & $12.187,78$ & 9 & $23.235,01$ & 7,25 \\
Quito & $12.051,52$ & 12 & $17.113,02$ & 16 & $18.958,79$ & 14 & $48.123,33$ & 15,02 \\
Ambato & $14.060,12$ & 14 & $10.695,63$ & 10 & $12.187,78$ & 9 & $36.943,53$ & 11,53 \\
Guayaquil & $3.012,88$ & 3 & 7.486 .94 & 7 & $6.770,99$ & 5 & $9.783,87$ & 3,05 \\
Otros & $2.008,59$ & 2 & $2.139,17$ & 2 & $8.125,18$ & 6 & $12.272,94$ & 3,83 \\
\hline Total & $100.429,37$ & 100 & $106.956,33$ & 100 & $135.419,80$ & 100 & $320.344,71$ & 100 \\
\hline
\end{tabular}

Fuente: Comité de Fiestas, Empresas Privadas, MINTUR

Los gastos para la realización de la fiesta de la Mama Negra en relación al origen de los proveedores reflejan que, para los tres periodos, el 59,30\% del presupuesto se invierte en la ciudad de Latacunga; en segundo lugar está la ciudad de Quito, a donde se destina el $15,02 \%$ del presupuesto; en tercer lugar está la ciudad vecina de Ambato, que acapara el $11,53 \%$ de la inversión, un importante $7,25 \%$ se destina hacia otros países por la contratación de espectáculos y artistas extranjeros; en algo más del 3\% del presupuesto se gasta en la ciudad Guayaquil, y cerca del $4 \%$ en otros sitios.

Esto quiere decir que en los tres años de análisis salieron de la ciudad de Latacunga y de la provincia de Cotopaxi hacia otras economías 130.358,68 dólares. Si el trabajo organizativo fuera más eficiente se podría lograr que un monto mayor a la mitad del presupuesto para la organización de la fiesta de la Mama Negra permaneciera en la ciudad de Latacunga y en la provincia de Cotopaxi.

\section{Impactos económicos indirectos}

Para el análisis del impacto indirecto se consideró la encuesta realizada a los turistas que pernoctaron en la ciudad de Latacunga, y el gasto promedio que realizaron durante su estadía, relacionado con la demanda explicita que está constituida por alojamiento, alimentación, transporte, compras de artesanías y souvenirs, localidades para espectáculos, costos de ingresos a centro de esparcimiento privados, entre otros.

Los resultados obtenidos indican que el gasto promedio por visitante es de $\$ 60,00$ dólares diarios, y el gasto promedio para una familia de cinco personas durante tres días de celebración es de 900 dólares; por ello, para obtener el impacto total se consideró la muestra de turistas derivada del reporte de pernoctaciones del MINTUR para cada año, el gasto promedio por turista, el número de días de pernoctación y el coeficiente de expansión.

Las preferencias de gasto promedio no variaron significativamente en los tres años analizados, ver tabla 4. Se obtiene un monto total de 2.154.557,37 dólares, pues, el aporte más significativo es de las actividades artísticas de entretenimiento y recreativas con 869.208,82, que equivale al 40,34\%; en segundo lugar se encuentran los ingresos por alojamiento y servicio de comidas con 719.275,71 dólares, que representa el 33,38\%; en tercer lugar se ubica el comercio al por mayor y menor con 387.556,58 dólares, equivalente al 17,99\%; el transporte y almacenamiento se ubican en cuarto lugar con 127.860,04 dólares, esto es el 5,93\%; y finalmente las actividades de industrias manufactureras que alcanzan 50.656,21 dólares en los tres años, que representa el 2,35\%. 
Tabla 4. Impacto indirecto en la economía local generado durante la fiesta de la Mama Negra en los periodos 2017, 2018, 2019

\begin{tabular}{|c|c|c|c|c|c|c|c|c|}
\hline \multirow{2}{*}{$\begin{array}{l}\text { Descripción de la } \\
\text { sección de Gasto }\end{array}$} & \multicolumn{2}{|c|}{ Año 2017} & \multicolumn{2}{|l|}{ Año 2018} & \multicolumn{2}{|c|}{ Año 2019} & \multicolumn{2}{|c|}{ Total, tres periodos } \\
\hline & Monto & $\%$ & Monto & $\%$ & Monto & $\%$ & Monto & $\%$ \\
\hline $\begin{array}{l}\text { Actividades } \\
\text { artísticas, de } \\
\text { entretenimiento y } \\
\text { recreativas }\end{array}$ & $267.476,74$ & 39 & $299.129,04$ & 42 & $302.603,04$ & 40 & $869.208,82$ & 40,34 \\
\hline $\begin{array}{l}\text { Actividades de } \\
\text { alojamiento y de } \\
\text { servicio de } \\
\text { comidas }\end{array}$ & $219.468,09$ & 32 & $235.029,96$ & 33 & $264.777,66$ & 35 & $719.275,71$ & 33,38 \\
\hline $\begin{array}{l}\text { Comercio al por } \\
\text { mayor y al por } \\
\text { menor; reparación } \\
\text { de vehículos } \\
\text { automotores y } \\
\text { motocicletas }\end{array}$ & $130.309,18$ & 19 & $121.076,04$ & 17 & $136.171,368$ & 18 & $387.556,58$ & 17,99 \\
\hline $\begin{array}{l}\text { Industrias } \\
\text { manufactureras }\end{array}$ & $13.716,75$ & 2 & $14.244,24$ & 2 & $22.695,22$ & 3 & $50.656,21$ & 2,35 \\
\hline $\begin{array}{l}\text { Transporte y } \\
\text { almacenamiento }\end{array}$ & $54.867,02$ & 8 & 42732,72 & 6 & $30.260,30$ & 4 & $127.860,04$ & 5,93 \\
\hline Total & $685.837,78$ & & 712.212 .00 & & 756.507 .60 & & $2.154 .557,37$ & 100,00 \\
\hline
\end{tabular}

Fuente: Encuestas realizadas a los turistas

\section{Medición del impacto inducido en la fiesta de la Mama Negra}

Como resultado del análisis de sostenibilidad turística de la fiesta de la Mama Negra se deduce que, la repercusión en la economía es positiva y alcanza un monto de 4.638.444,97 dólares en los tres años de análisis.

Las actividades con mayor representatividad es la artística y de entretenimiento que ha generado 1.969.018 dólares, que representa el $42.45 \%$ de los ingresos; el segundo rubro en importancia para la sostenibilidad de la economía del turismo por la festividad de la Mama Negra fue la actividad vinculada al alojamiento y servicios de comida que ha alcanzado para los tres años de evaluación 1.301.237,83 dólares, estos es, 28,05\%; el tercer rubro en importancia producto de la medición del impacto económico inducido fue el comercio al por mayor y menor con 622.016,66 dólares, logrando un 13,41\%; en cuarto lugar se ubican las industrias manufactureras de la ciudad de Latacunga con 413.254,21 dólares, que equivale a 8,91\%; es importante anotar el rubro de transporte y almacenamientos que alcanza 274.956,15 dólares, que significa el 5,93\%; los demás rubros se encuentran bajo el $1 \%$, pero se les podría activar para que se integren con más fuerza en la generación de ingresos. El desglose de la medición del impacto inducido se puede ver en la tabla 5 . 
Tabla 5. Impacto inducido del desarrollo de la fiesta de la Mama Negra periodos 2017, 2018, 2019

\begin{tabular}{|c|c|c|c|c|c|c|c|}
\hline $\begin{array}{c}\text { Descripción de la sección de } \\
\text { Gasto }\end{array}$ & $\begin{array}{l}\text { Impacto } \\
\text { directo }\end{array}$ & $\begin{array}{l}\text { Impacto } \\
\text { Indirecto }\end{array}$ & $\begin{array}{l}\text { Total, de } \\
\text { Impacto }\end{array}$ & $\begin{array}{l}\text { Multiplicadores } \\
\text { Sectores Utilizados }\end{array}$ & Multiplicador & $\begin{array}{l}\text { Impacto } \\
\text { Inducido }\end{array}$ & $\%$ \\
\hline $\begin{array}{l}\text { Actividades artísticas, de } \\
\text { entretenimiento y recreativas }\end{array}$ & 224690,5 & $869.208,82$ & $1.093 .899,30$ & $\begin{array}{l}\text { Industrias del } \\
\text { turismo }\end{array}$ & 1,8 & $1.969 .018,74$ & 42,5 \\
\hline $\begin{array}{l}\text { Actividades de alojamiento y } \\
\text { de servicio de comidas }\end{array}$ & 3634,2 & $719.275,71$ & $722.909,91$ & $\begin{array}{l}\text { Hoteles y } \\
\text { restaurantes }\end{array}$ & 1,8 & $1.301 .237,83$ & 28,1 \\
\hline $\begin{array}{l}\text { Actividades de servicios } \\
\text { administrativos y de apoyo }\end{array}$ & 6183,12 & 0 & $6.183,12$ & $\begin{array}{l}\text { Otros servicios } \\
\text { sociales y } \\
\text { personales }\end{array}$ & 1,2 & $7.419,74$ & 0,16 \\
\hline $\begin{array}{l}\text { Actividades financieras y de } \\
\text { seguros }\end{array}$ & 458,43 & 0 & 458,43 & $\begin{array}{l}\text { Financiación de } \\
\text { planes de seguros y } \\
\text { de pensiones. }\end{array}$ & 2,2 & $1.008,55$ & 0,02 \\
\hline Actividades inmobiliarias & 17176,05 & 0 & $17.176,05$ & Alquiler de vivienda & 1,3 & $22.328,86$ & 0,48 \\
\hline $\begin{array}{l}\text { Administración pública y } \\
\text { defensa; planes de seguridad } \\
\text { social de afiliación } \\
\text { obligatoria }\end{array}$ & 6202,95 & 0 & $6.202,95$ & $\begin{array}{l}\text { Administración } \\
\text { pública y defensa; } \\
\text { Seguro obligatorio }\end{array}$ & 1,5 & $9.304,43$ & 0,2 \\
\hline $\begin{array}{l}\text { Comercio al por mayor y al } \\
\text { por menor; reparación de } \\
\text { vehículos automotores y } \\
\text { motocicletas }\end{array}$ & 1203,82 & $387.556,59$ & $388.760,41$ & $\begin{array}{l}\text { Comercio al por } \\
\text { mayor y al } \\
\text { por menor }\end{array}$ & 1,6 & $622.016,66$ & 13,4 \\
\hline Industrias manufacturas & 67416,42 & $50.656,21$ & $118.072,63$ & $\begin{array}{l}\text { Industrias } \\
\text { manufactureras }\end{array}$ & 3,5 & $413.254,21$ & 8,91 \\
\hline $\begin{array}{l}\text { Información y } \\
\text { comunicaciones }\end{array}$ & 12768,57 & & $12.768,57$ & $\begin{array}{l}\text { Correos y } \\
\text { telecomunicaciones }\end{array}$ & 1,4 & $17.899,80$ & 0,39 \\
\hline Transporte y almacenamiento & 3071,46 & $127.860,04$ & $130.931,50$ & $\begin{array}{l}\text { Transporte y } \\
\text { almacenamiento }\end{array}$ & 2,1 & $274.956,15$ & 5,93 \\
\hline Total & 342805,5 & $2.154 .557,37$ & 2497362,87 & & & $4.638 .444,97$ & 100 \\
\hline
\end{tabular}

\section{Aspectos relevantes que influyeron en la sostenibilidad turística de la fiesta de la Mama Negra}

A continuación, se analizan algunos indicadores del perfil del turista que influyen indirectamente en la sostenibilidad económica del turismo en la festividad de la Mama Negra y que no han sido considerados en el análisis previo, entre ellos los más sobresalientes son los siguientes:

Los turistas que pernoctaron en la ciudad de Latacunga y que llegaron específicamente para disfrutar de la fiesta de la Mama Negra proceden de las provincias de Pichincha el $40 \%$, de Tungurahua el $20 \%$, de otros cantones de la provincia de Cotopaxi el $12 \%$, de otras provincias de la sierra el 9\%, de la provincia de Chimborazo el 6\%, de otras provincias de la costa el 5\%, de la provincia del Guayas el 4\%, turistas extranjeros el 3\% y procedentes de la Amazonía ecuatoriana 3\%, ver figura 1. 


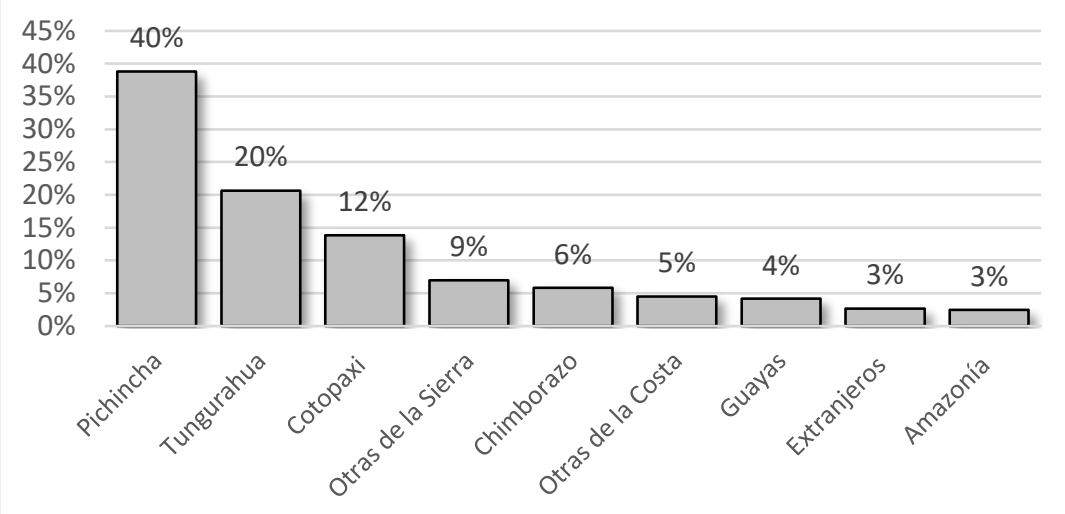

Figura 1. Procedencia de los Turistas

Fuente: Encuestas.

El grafico de barras demuestra que la mayor cantidad de turistas provienen de las dos provincias vecinas, de Pichincha y Tungurahua con énfasis en la primera. Se observa además una importante presencia de turistas de la provincia de Chimborazo, y un porcentaje disperso entre las demás provincias de la Sierra, Costa y Amazonía. Es importante también la presencia de turistas de otros cantones de la provincia de Cotopaxi, demostrando que la fiesta de la Mama Negra atrae a turistas de todos los sitios del Ecuador. Se ha encontrado un bajo porcentaje de turistas extranjeros, lo que indica la falta de promoción de la festividad internacionalmente. Estos datos contribuyen a la sostenibilidad, pues, indican la orientación que se debe tener para mejorar la calidad de servicios de acuerdo con las preferencias de los visitantes, lo que generara mayores ingresos.

Los turistas que llegan al destino de la ciudad de Latacunga a disfrutar de las festividades de la Mama Negra se transportan de diversa manera, pues, el $43 \%$ se traslada en automóvil propio, lo que les permite ampliar el recorrido conociendo otros atractivos de la provincia de Cotopaxi, esto implica el respectivo consumo de gasolina para el traslado; un 52\% se movilizan en transporte público utilizando unidades de buses de rutas interprovinciales con capacidad de entre 35 y 45 pasajeros; solo el 5\% de los turistas llegan a la ciudad en unidades de transporte alquiladas o en taxis, ver figura 2. Por lo que, el transporte público tiene un aumento significativo; $y$, por consiguiente, mejoran también sus ingresos.

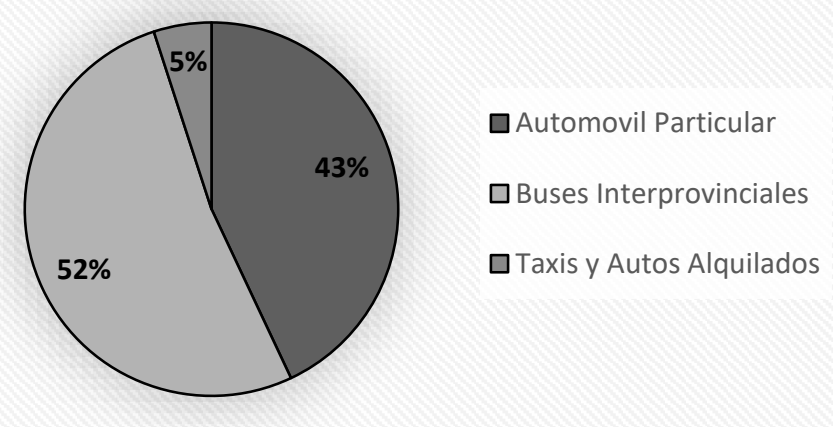

Imagen 2. Medios de transporte Utilizados por los turistas Fuente: Encuestas 
En cuanto a las actividades organizadas que prefieren los turistas en su periodo de estadía en la ciudad de Latacunga por las festividades de la Mama Negra, las de mayor demanda fueron los desfiles $26 \%$, eventos culturales $21 \%$, eventos feriales $20 \%$, recorridos urbanos $11 \%$, recorridos provinciales $9 \%$, conciertos y espectáculos nocturnos $7 \%$, bares y discotecas 6\% (Figura 3).

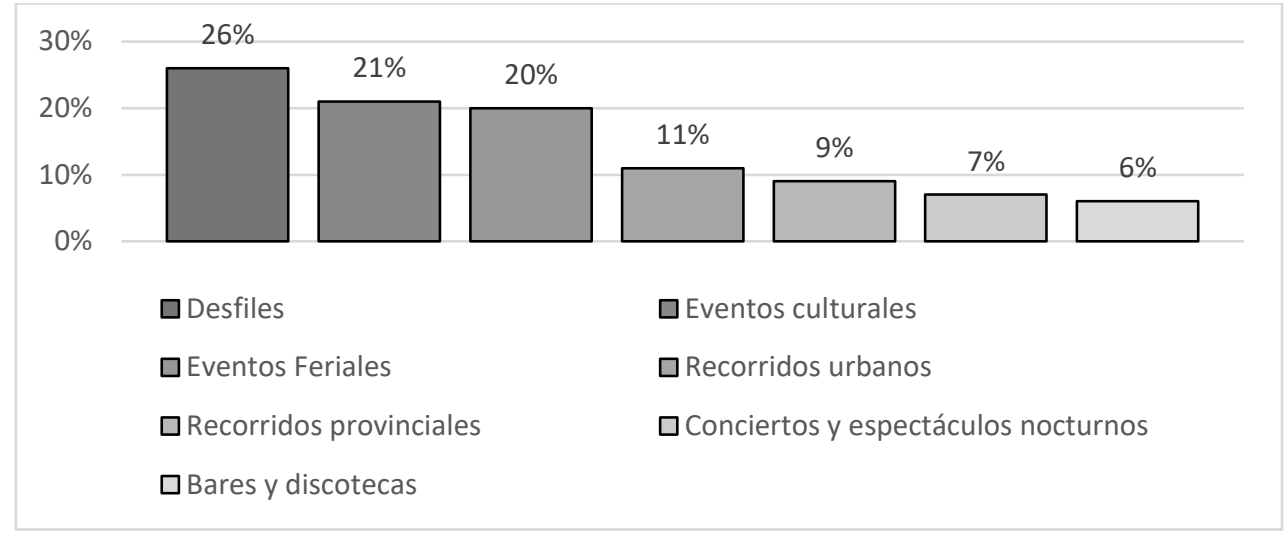

Imagen 3. Actividades organizadas que prefieren los turistas

Fuente: Encuestas

Los datos precedentes indican algunas preferencias de los turistas; por tanto, son afirmaciones que se consideran importantes para estimular y potenciar aquellas áreas en las que se podría obtener una ventaja competitiva y ser aprovechadas de mejor manera. Resulta evidente que la principal atracción es el desfile o cabalgata de la Mama Negra, sin embargo, se pueden observar la intención de los visitantes por participar en eventos culturales y ferias, espacios en los que se puede mejorar la oferta de productos de la pequeña empresa como artesanías, conservas, alimentos, entre otros. Es importante también el deseo de los turistas por realizar recorridos urbanos y en otros lugares de la provincia, entendiéndose que la festividad de la Mama Negra es una oportunidad para otros sectores productivos y áreas geográficas de la provincia de Cotopaxi y la región Sierra Centro.

También se proponen a continuación algunas sugerencias expuestas por los turistas que, al ser consideradas podrían fortalecer las acciones para dar sostenibilidad económica a la actividad turística de la festividad de la Mama Negra en la ciudad de Latacunga. Sobre la infraestructura y la promoción de servicios, los visitantes sugieren que se debe promocionar de mejor manera los servicios hoteleros y restaurantes, y algo muy importante, que se eleve la calidad de los servicios capacitando al personal y que la infraestructura se ajuste a las necesidades de los visitantes.

Sobre la gestión de la fiesta de la Mama Negra que deben realizar los organizadores del evento, los visitantes proponen las siguientes sugerencias: que se mejore el tránsito en la ciudad creando vías alternas para dar mayor fluidez al tráfico y se creen más espacios para aparcar los automotores con la garantía de trato y seguridad. 
También proponen que se mejore las condiciones del desfile con mayor fluidez, menos interrupciones, menor número de vendedores ambulantes, respetando los horarios; para ello, es importante que se busquen los mecanismos para que la ciudadanía y los visitantes puedan apreciar las comparsas con mayor facilidad, pues, podría ser una alternativa para dar sostenibilidad económica a los habitantes de la ciudad de Latacunga.

Asimismo, señalan que se debe trabajar organizadamente para mantener limpia la ciudad, mediante campañas de limpieza con la población, y mejorando las condiciones de trabajo de los obreros municipales encargados de la limpieza, para ello, la población deberá contribuir operativamente con este proceso.

Se sugiere mejorar la promoción y difusión de la festividad de la Mama Negra a nivel nacional, y difundir la programación de fiestas a los habitantes de la ciudad de Latacunga, para que sean ellos los agentes de divulgación de los eventos turísticos. Al respecto, los visitantes solicitan que se realicen mayor cantidad de espectáculos populares y al mismo tiempo que se eleve la calidad, así como de los artistas.

Finalmente, los turistas proponen un mayor control de parte de las autoridades a la sociedad civil, bajando el consumo de alcohol, disminuyendo los niveles de delincuencia, mejorando la seguridad en el tránsito, controlando la especulación sobre todo de los servicios de alojamiento y alimentación, y promoviendo el respeto a la integridad y diversidad de los turistas.

\section{Discusión}

La sostenibilidad y competitividad de destinos turísticos en la actualidad se entiende como el desarrollo de la oferta de bienes y servicios, sin arriesgar la viabilidad de los recursos naturales, sociales y culturales. Al respecto García, Macias, Jurado \& Bonaño (2017), consideran que la sostenibilidad económica vinculada a la actividad turística, lo que busca es implementar un nuevo modelo de consumo y producción turística que considere el cuidado de los recursos turísticos.

Desde la perspectiva de esta investigación se considera que la sostenibilidad es una condición necesaria que permite tomar decisiones para promover el desarrollo turístico en un espacio geográfico determinado, considerando el nivel de afectación que esta actividad es capaz de generar. Entonces, la evaluación de la sostenibilidad en el turismo es fundamental para establecer los factores intervinientes del capital turístico, y que se componen de los recursos sinérgicos que interactúan en el destino, por lo que, se puede establecer que la sostenibilidad del turismo dependerá de la forma en la que se mantengan y renueven los elementos de este patrimonio territorial.

Al respecto Rojo (2018), asegura que el turismo sostenible aspira a ser una actividad económicamente viable, que al tiempo evita destruir los recursos de los que el propio turismo dependerá en el futuro, principalmente en lo que afecta al entorno físico y a la estructura social de la comunidad local. Este análisis lleva a pensar que toda argumentación sobre la industria turística, así como los procesos de organización y 
planificación deben ser inseparables de las siguientes tres dimensiones: económica, social y ambiental.

Las razones que motivan este trabajo de investigación son la económica y social vinculadas al turismo, como una forma de crear espacios de desarrollo principalmente enfocando a la actividad turística asociada al patrimonio cultural inmaterial como una oportunidad de generar fuentes de trabajo, esta quizá será en un futuro cercano la contribución del turismo al Ecuador. Al respecto existe una importante evidencia como en el caso de la fiesta de la Mama Negra en la ciudad de Latacunga, muestra el papel relevante de las fiestas populares en su contribución para el mejoramiento de las condiciones económicas de los habitantes de un territorio.

Telfer \& Sharpley (2016), aseguran que las actividades turísticas de relevancia en un territorio están vinculadas al servicio de hospedaje y alimentación, así como también aquellas dedicadas a la recreación y al transporte, así como a los grupos que hacen posible la operación turística. Estas empresas han demostrado un crecimiento importante en los últimos años, un ejemplo de ello son las multinacionales de turismo. En los países en desarrollo la industria hotelera es todavía incipiente, sin embargo, juegan un papel fundamental en la concentración del desarrollo turístico local, poniendo un énfasis especial en la capacidad hotelera instalada en las ciudades en las que se tienen eventos importantes una vez al año, época en la que se sobresatura la demanda. Mientras que durante el resto del año esta infraestructura pasa semi-ociosa; en este sentido, se debe considerar la activación de un turismo que oferte actividades durante todo el año y que relacionadas con la fecha principal, esto con el propósito de dar mayor sostenibilidad económica a este segmento de recursos turísticos que podría representar una alternativa de desarrollo importante. El turismo puede diversificarse, requiere de iniciativas creativas sobre todo en los sitios en los que se ha consolidado una fiesta patrimonial reconocida a nivel nacional e internacional, no puede ser posible asegura Correrá (2015), que los recursos existentes se subutilicen, por lo que es fundamental abrir espacios y motivar iniciativas.

La concreción de la sostenibilidad económica fundamentada en el turismo requiere de la participación eficiente de los actores y ejecutores de la actividad turística en los territorios, pues, el turismo no puede ni debe ser considerado como una alternativa de segundo orden para el desarrollo económico, sino, como parte del engranaje socio económico en el contexto de las economías emergentes (Castro, 2019).

En esta investigación se establece que a partir de un análisis económico racional, las ventajas que pueden obtener los habitantes de una localidad cuando existe el compromiso para la promoción de un evento que atrae a un importante número de visitantes por sus características sociales, culturales y patrimoniales, y que se puede vincular con otras actividades de carácter recreativo para dar lugar a una fiesta de connotación nacional que es capaz de convocar cada año a cerca de 300.000 personas, y generar un promedio aproximado de 1.500 .000 dólares, redundan en beneficio de la población. 
Para terminar, el desarrollo de esta discusión invita a reflexionar sobre las capacidades locales para potenciar los recursos turísticos de los territorios, teniendo como base eventos de reconocimiento nacional que se circunscriben como parte del patrimonio inmaterial de la nación y sobre los cuales se tiene montada infraestructura y capacidad operativa excepcional. La pregunta es entonces, ¿Cuáles son los elementos de planificación y organización que se requieren para generar actividades colaterales que redunden en la sostenibilidad económica de los territorios potencializando los recursos turísticos?

\section{Conclusiones}

- La sostenibilidad económica del turismo en el Ecuador tiene como referente espacios en los que se realizan grandes festejos en determinadas épocas del año; sin embargo, estas actividades son estacionales, pues, el calendario festivo implica una serie de eventos cívicos, religiosos y culturales que abarcan a todo el territorio nacional y no dejan espacios abiertos.

- Para albergar a los turistas en determinadas fechas del año, se crea capacidad instalada turística en las diferentes ciudades del país para uno o dos eventos, como es el caso de la Mama Negra en Latacunga, la fiesta de las Flores y las Frutas en Ambato, el Carnaval de Guaranda, la Diablada de Píllaro, entre otras. Esto hace que dicha capacidad se mantenga ociosa por mucho tiempo, pues, son escasos los territorios que reciben regularmente durante todo el año a los viajeros.

- En este trabajo se demuestra que las actividades turísticas generadas desde las festividades populares como el caso de la Mama Negra, son un espacio de desarrollo económico muy importante para las comunidades locales durante el periodo de tiempo en el que se lleva a cabo, pues, permite un desarrollo económico superior al resto del año.

- El estudio de sostenibilidad económica del turismo para la festividad de la Mama Negra implicó un análisis de los impactos económicos directos e indirectos; así mismo, se estableció un coeficiente mecánico para determinar una aproximación de los ingresos globales que tuvo la ciudad de Latacunga por efecto de la realización de dicha festividad.

- Se logró evidenciar la importancia de la fiesta de la Mama Negra como factor de sostenibilidad económica del turismo en la ciudad y provincia, actividad que integra la participación de actores públicos y privados que se articulan para dar realce a una de las principales manifestaciones inmaterial del Ecuador.

\section{Referencias bibliográficas}

Álvarez, F. (2015). Capital social, sinergia, impacto social y las organizaciones de la sociedad civil. Realidad y Reflexión, 15(41), 8-27.

Blasco, J., Martínez, S., \& Alonso, M. (2017). El Patrimonio como elemento dinamizador de la socioeconomía de las comunidades locales en el marco de las políticas europeas. Propuesta de acciones y actuaciones turísticas. Boletín de la 
Asociación de Geografos Españoles(73), 413-429. doi:DOI:

$10.21138 /$ bage. 2424

Castro L, J. (2018). La Planeación sostenible de ciudades: propuestas para el desarrollo de infraestructuras. México: Fondo de Cultura Económica.

Castro, A. (2019). Contradicciones entre turismo, economía y ecología. Revista Economía y Desarrollo, 134(2).

Chica, U. (2019). Análisis semiótico de la vestimenta de los personajes de la fiesta de la Mama Negra. Cuenca: Universidad del Azuay.

Corrales, L. (2019). El personaje de la Mama Negra com representación social y su simbología en la fiesta de la Santísima tragedia o capitanía realizada en septiembre en Latacunga. Ambato: Universidad Tecnica de Ambato.

Correra, A. (2015). El impacto económico del turismo accesible en Europa. Rstúdios Turísticos, 203, 27-37.

Cortés, F. (2008). Selección no aleatoria y validez a propósito de la evaluación cualitativa de oprtunidades. Método Científico Y Política Social, 62-81.

Cuervo, G. (2017). Desarrollo económico local: leyendas y Realidades. Territorios, 1, 9-24.

Cuvi, P. (2005). Las Fiestas Populares en el Ecuador. Quito: Ministerio de Turismo.

Eddy, A. (2016). Impacto económico del turismo en la economía del Ecuador. Revista Anais Brasileiros de Estudos Turístico ABET, 69-81.

Escudero, S. (2017). Las Fiestas populares en el Ecuador: Un factor de interacción comunitaria. Revista Universidad y Sociedad, 9(3), 27-33.

GAD Municipal de Latacunca . (2016). Plan de Ordenamiento Territorial del Cantón Latacunga. Latacunga: Gad Latacunga.

García, F., Macías, R., Jurado, E., \& Bonaño, J. (2017). Sostenibilidad y competitividad en Desrtinos Turísticos. Iafet Cognocere, 2-10. Obtenido de http://www.academia.edu/download/44401423/Congreso_OMT_Sostenibilidad_ y_competitividad_destinos.pdf

Giacomotti, G. (19 de Septiembre de 2019). Latacunga reactiva su economía con la fiesta de la Mama Negra. El Comercio, pág. Digital.

Güell, J. (2019). Planificación Estartégica de Ciudades: Nuevos Instrumentos y Procesos. Barcelona: Reverte.

Herner, M. (2016). Patrimonio cultural inmaterial y turismo: fiestas populares como organizadoras del territorio. Entrevistas: Revista de Debates, 8(7), 1-11. 
Herrero, L. (2004). Impacto Económico de los Macro-Festivales Culturales: Reflexiones y Resultados. Universidad de Valladolid. Valladolida: Boletín GC Cultural.

Maldonado, W., Arúz, B., \& Pinos, D. (2017). Propuesta metodológica de evaluación del balance social en asociaciones de economía popular y solidaria del Ecuador. CIRIEC_España, revista de economía pública, social y cooperativa, 90, 123157.

Méndez , R. (2016). Formulación y evaluación de proyectos. Enfoque para emprendedores. Entornos, 475-478.

MINTUR. (2019). Servicios Turísticos, estadísticos de oferta. Obtenido de EStablecimientos Registrados : https://servicios.turismo.gob.ec/index.php/turismo-cifras/2018-09-18-21-1117/establecimientos-registrados

Montes, F., \& Cantuche, M. (2005). Modelos input-output y análisis probabilístico de impacto cruzado mediante escenarios. Revista de Economía Mundial, 13, 99112.

Olivera, A., Cristobal, S., \& Saizar, C. (2016). Análisis de ciclo de vida ambiental, económico y social. Una herramienta para la evaluación de impactos y soporte para la toma de decisiones. Innotec gestión , 20-27.

Oyardi, R., Nazareno, V., Roldán, R., \& Ferrales, A. (2016). Emprendimiento como factor del desarrollo turístico rural sostenible. Retos de la Dirección, 10(1), 7193.

Pearce, G. (2016). Modelos de gestión de destinos. Sintesis y evaluación. Estudios y persepectivo e turismo, 25(1), 1-16.

Perea, M., Navarro, J., \& Luque, G. (2018). Inteligencia Territorial: Conceptualización y aance en el estado de la cuestión. Vínculos posibles con los destinos turísticos. Cuadernos de Turísmo, 535-554. doi:DOI: http://dx.doi.org/10.6018/turismo.41.327141

Picornell, C. (2015). Los impactos del turismo. Papers de turisme, 11, 65-91.

Plasencio, O. (2015). "Promoción y Difusión de las Reminiscencias, Costumbres, Tradiciones de la Fiesta de la Mama Negra en la ciudad de Latacunga. Ambato: UNIANDES.

Polanco, C. (2006). Indicadores ambientales y modelos internacionales para la toma de desiciones. Gestión y Ambiente, 9(2), 27-41.

Proaño, R. (2019). Caracterización de las fiestas populares de la provincia de Cotopaxi y su aporte histórico al turismo: caso fiestas de la mama negra. Ambato: UNIANDES. 
Ramírez , Y. (2015). Las fiestas populares tradicionales, reflejo de la identidad cultural de las comunidades. Revista Caribeña de Ciencias Sociales, 5-12.

Rojo M, I. (2018). Gestión sostenible de empress y dstinos turísticos: La NUeva Economía . Responsabilidad social de la empresa , 103-112.

Ruíz , L., \& García , L. (2014). Análisis de la falla ambiental de las construcciones turísticas en ecosistemas costeros. Cuba: Medio Ambiente y Desarrollo. Revista Electrónica de la Agencia de Medio Ambiente, 14(16). Obtenido de https://www.academia.edu/10509722/

Soto, J. (2015). El crecimiento urbano de las ciudades: enfoques desarrollista, autoritario, neoliberal y sustentable. PAradigma económico, 7(1), 127-149.

Telfer J, D., \& Sharpley, R. (2016). Tourism and Development in the Developing World. New York : Routledge.

Torres, J., Ullauri, N., \& Lalangui, J. (2018). Las celebraciones andinas y fiestas populares como identidad ancestral del Ecuado. Revista Universidad y Sociedad, 10(2), 294-303.

Vaca, V. (2016). Compendio colaborativo de la Mama Negra. Quito: USFQ.

Wassilowsky A, H. (2017). Turismo patrimonial, identidad y desarrollo en el Perú. Indiana, 34(1), 199-230.

Zambrano, R. (2015). La teatralidad en la fiesta popular La Mama Negra. Quito: UCE.

\section{Ciencia Ligital}




\section{PARA CITAR EL ARTÍCULO INDEXADO.}

Silva Viteri, X. A., Auquilla Belema, L. A., Fernández Sánchez, L. del R., \& Auquilla Ordóñez, Álvaro A. (2020). Sostenibilidad económica del turismo en el Ecuador: caso festividad de la "Mama Negra" Latacunga. Explorador Digital, 4(4), 27-49. https://doi.org/10.33262/exploradordigital.v4i4.1413

\section{LC Ciencia}

El artículo que se publica es de exclusiva responsabilidad de los autores y no necesariamente reflejan el pensamiento de la Revista Explorador Digital.

El artículo queda en propiedad de la revista y, por tanto, su publicación parcial y/o total en otro medio tiene que ser autorizado por el director de la Revista Explorador Digital.
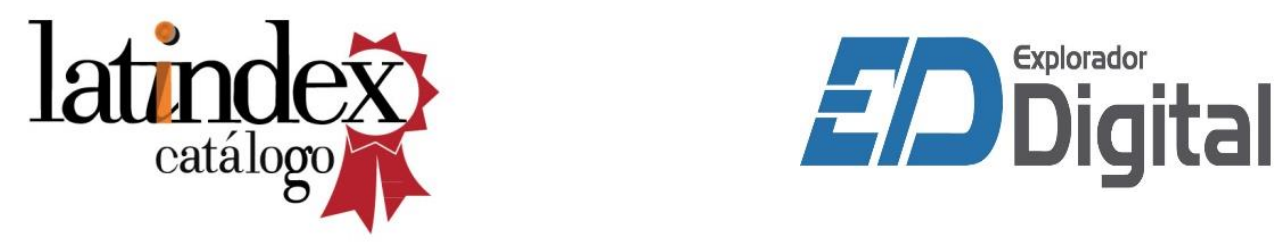